\title{
Hyperbolic rank and subexponential corank of metric spaces
}

\author{
Buyalo, S ; Schroeder, Viktor
}

\begin{abstract}
We introduce a new quasi-isometry invariant corank X of a metric space X called subexponential corank. A metric space $\mathrm{X}$ has subexponential corank $\mathrm{k}$ if roughly speaking there exists a continuous map , $\mathrm{T}$ is a topological space, such that for each the set $\mathrm{g}-1(\mathrm{t})$ has subexponential growth rate in $\mathrm{X}$ and the topological dimension $\operatorname{dim} \mathrm{T}=\mathrm{k}$ is minimal among all such maps. Our main result is the inequality for a large class of metric spaces $\mathrm{X}$ including all locally compact Hadamard spaces, where rank $\mathrm{h} \mathrm{X}$ is the maximal topological dimension of among all CAT $(-1)$ spaces $\mathrm{Y}$ quasi-isometrically embedded into $\mathrm{X}$ (the notion introduced by M. Gromov in a slightly stronger form). This proves several properties of rank $\mathrm{h}$ conjectured by Gromov, in particular, that any Riemannian symmetric space $\mathrm{X}$ of noncompact type possesses no quasi-isometric embedding of the standard hyperbolic space $\mathrm{H} n$ with $\mathrm{n}-1>\operatorname{dim} \mathrm{X}$ - rank X.
\end{abstract}

DOI: https://doi.org/10.1007/s00039-002-8247-7

Posted at the Zurich Open Repository and Archive, University of Zurich

ZORA URL: https://doi.org/10.5167/uzh-21934

Journal Article

Accepted Version

Originally published at:

Buyalo, S; Schroeder, Viktor (2002). Hyperbolic rank and subexponential corank of metric spaces. Geometric and Functional Analysis, 12(2):293-306.

DOI: https://doi.org/10.1007/s00039-002-8247-7 


\title{
Hyperbolic rank and subexponential corank of metric spaces
}

\author{
Sergei Buyalo* \& Viktor Schroeder
}

\begin{abstract}
We introduce a new quasi-isometry invariant $\operatorname{corank} X$ of a metric space $X$ called subexponential corank. A metric space $X$ has subexponential corank $k$ if roughly speaking there exists a continuous map $g: X \rightarrow T$ such that for each $t \in T$ the set $g^{-1}(t)$ has subexponential growth rate in $X$ and the topological dimension $\operatorname{dim} T=k$ is minimal among all such maps. Our main result is the inequality $\operatorname{rank}_{h} X \leq \operatorname{corank} X$ for a large class of metric spaces $X$ including all locally compact Hadamard spaces, where $\operatorname{rank}_{h} X$ is maximal topological dimension of $\partial_{\infty} Y$ among all CAT $(-1)$ spaces $Y$ quasi-isometrically embedded into $X$ (the notion introduced by M. Gromov in a slightly stronger form). This proves several properties of $\mathrm{rank}_{h}$ conjectured by M. Gromov, in particular, that any Riemannian symmetric space $X$ of noncompact type possesses no quasi-isometric embedding $\mathrm{H}^{n} \rightarrow X$ of the standard hyperbolic space $\mathrm{H}^{n}$ with $n-1>\operatorname{dim} X-\operatorname{rank} X$.
\end{abstract}

\section{Introduction}

Given a metric space $X$ consider all locally compact CAT(-1) Hadamard spaces $Y$ quasi-isometrically embedded into $X$ and let

$$
\operatorname{rank}_{h} X=\sup _{Y} \operatorname{dim} \partial_{\infty} Y
$$

over all such $Y$. This quasi-isometry invariant is introduced in [Gr, 6. $\left.\mathrm{B}_{2}\right]$ in a slightly stronger form requiring only $Y$ to be a geodesic hyperbolic space, where it is called hyperbolic corank. We prefer to call it hyperbolic rank reserving the term corank for a dual notion which is central for this paper. We have $\operatorname{rank}_{h} X=\operatorname{dim} \partial_{\infty} X$ for each CAT(-1) space $X$ (this easily follows from the Morse' quasi-isometry lemma) and it is known that the Cartesian product $X=X_{1} \times \cdots \times X_{k}$ of Hadamard manifolds with pinched negative curvature, $-k^{2} \leq K \leq-1$, always has

$$
\operatorname{rank}_{h} X \geq \sum_{i=1}^{k} \operatorname{rank}_{h} X_{i}
$$

*Supported by RFFI Grants 99-01-00104, 00-15-96024 and SNF Grant 20-57151.99 
(see $[\mathrm{Gr}, \mathrm{BF}]$ for $X_{i}$ real hyperbolic manifolds and [FS] for the general case). Next, let $X$ be a Riemannian symmetric space of noncompact type. Then

$$
\operatorname{rank}_{h} X \geq \operatorname{dim} X-\operatorname{rank} X
$$

(see $[\mathrm{Le}]$ ). It is conjectured in $[\mathrm{Gr}]$ that there is equality in both cases.

We prove these conjectures by introducing a new quasi-isometry invariant corank $X$ of a metric space $X$ called subexponential corank of $X$ and by showing that $\operatorname{rank}_{h} X \leq \operatorname{corank} X$ for each "reasonable" $X$. A metric space $X$ has subexponential corank $k$, corank $X=k$, if roughly speaking there exists a continuous map $g: X \rightarrow T$ such that for each $t \in T$ the set $g^{-1}(t)$ has subexponential growth rate in $X$ and the topological dimension $\operatorname{dim} T=k$ is minimal among all such maps (for the precise definition see sect. 2). In this case, we say that $X$ supports a subexponential partition of rank $k$. One easily sees that $\operatorname{corank}\left(X_{1} \times X_{2}\right) \leq \operatorname{corank} X_{1}+\operatorname{corank} X_{2}$, that corank $X \leq \operatorname{dim} X-1$ for every Hadamard manifold $X$ and that corank $X \leq \operatorname{dim} X-\operatorname{rank} X$ for every Riemannian symmetric space of noncompact type.

The following theorem, which is our main result, immediately implies the above conjectures. Moreover, it works in both directions establishing obstructions as to quasi-isometric embeddings of CAT $(-1)$ spaces into $X$ as well as to the existence of subexponential partitions of $X$.

We say that a metric space $X$ has the $Q P C$ property or $X$ is a QPC-space if every quasi-isometric map $f: Y \rightarrow X$ is parallel to a continuous one, i.e., there exists a continuous $f^{\prime}: Y \rightarrow X$ with $\operatorname{dist}\left(f^{\prime}(y), f(y)\right) \leq C<\infty$ for all $y \in Y$ (QPC stays for Quasi-isometric maps are Parallel to Continuous ones). For example, every locally compact Hadamard space is QPC (for more details see sect. 2).

Theorem 1.1. Let $X$ be a metric space which is quasi-isometric to a $Q P C$ one. Then

$$
\operatorname{rank}_{h} X \leq \operatorname{corank} X .
$$

In particular, there is no quasi-isometric embedding $Y \rightarrow X$ of $\mathrm{CAT}(-1)$ spaces $Y$ with $\operatorname{dim} \partial_{\infty} Y>\operatorname{corank} X$ and if $X$ is $Q P C$ then it supports no subexponential partition of rank less than $\operatorname{rank}_{h} X$.

From [BF, FS, Le] and Theorem 1.1 we obtain

Corollary 1.2. (1) Let $X$ be the Cartesian product of Hadamard manifolds with pinched negative curvature, $X=X_{1} \times \cdots \times X_{k}$. Then

$$
\operatorname{rank}_{h} X=\sum_{i=1}^{k} \operatorname{rank}_{h} X_{i}=\sum_{i=1}^{k} \operatorname{corank} X_{i}=\operatorname{corank} X .
$$

(2) Let $X$ be a Riemannian symmetric space of noncompact type. Then

$$
\operatorname{rank}_{h} X=\operatorname{dim} X-\operatorname{rank} X=\operatorname{corank} X .
$$


Remark 1.3. We use the CAT $(-1)$ condition in the definition of $\operatorname{rank}_{h}$ to simplify the proof of Theorem 1.1. It can be modified in a way to include all (complete, locally compact) geodesic hyperbolic spaces proving by that Gromov's conjectures in their full generality.

The invariant corank and Theorem 1.1 have further applications. For instance, we prove

Theorem 1.4. Let $X$ be the metric universal covering of a closed 3dimensional nonpositively curved graph manifold. Then corank $X \leq 1$. As a consequence, $Z=X_{1} \times \cdots \times X_{n}$ has corank $Z \leq n$ and possesses no quasiisometric embedding $Y \rightarrow Z$ of $\mathrm{CAT}(-1)$ spaces $Y$ with $\operatorname{dim} \partial_{\infty} Y>n$, where each $X_{i}$ is the universal covering of a 3-dimensional nonpositively curved graph manifold.

Note that each $X_{i}$ in Theorem 1.4 is not a $\operatorname{CAT}(-1)$ space. It only possesses a quasi-isometric embedding $\mathrm{H}^{2} \rightarrow X_{i}$, hence $Z=X_{1} \times \cdots \times X_{n}$ has an exponential growth rate and $\operatorname{rank}_{h} Z=n$ by combining the results of $[\mathrm{BF}]$ and Theorem 1.4.

Another consequence of Theorem 1.1 is that $\operatorname{rank}_{h} X=n-1=\operatorname{corank} X$ for every $\mathrm{CAT}(-1)$ Hadamard manifold $X, \operatorname{dim} X=n$. One might expect that the equality $\operatorname{rank}_{h} X=\operatorname{corank} X$ is true for each CAT(-1) space $X$. However, this is not the case. For example, let $X$ be a simplicial tree whose vertices have degree at least 3 and whose edges have length 1 . Then $X$ is a CAT $(-1)$ space with $\operatorname{rank}_{h} X=\operatorname{dim} \partial_{\infty} X=0$ while corank $X=1$ because every continuous map $X \rightarrow T$ into 0 -dimensional space $T$ is constant and $X$ has exponential growth rate.

We conclude the Introduction by a sketch of our initial proof that there is no quasi-isometric embedding $f: \mathrm{H}^{3} \rightarrow \mathrm{H}^{2} \times \mathbb{R}^{n}$ for any $n \geq 0$. This proof was the starting point to introduce the subexponential corank and finally to prove Theorem 1.1. Note that any simple counting argument does not work because both the source space $\mathrm{H}^{3}$ and the target space $\mathrm{H}^{2} \times \mathbb{R}^{n}$ have exponential growth rates.

Assume that there is such an $f$. Assume for simplicity that $f$ is smooth and biLipschitz, that is $\frac{1}{a} \operatorname{dist}\left(x, x^{\prime}\right) \leq \operatorname{dist}\left(f(x), f\left(x^{\prime}\right)\right) \leq a \operatorname{dist}\left(x, x^{\prime}\right)$ for some $a \geq 1$ and all $x, x^{\prime} \in \mathrm{H}^{3}$. Fix a horosphere $T \subset \mathrm{H}^{2}$ and define a map $g: \mathrm{H}^{2} \times \mathbb{R}^{n} \rightarrow T$ by projecting onto the first factor and then projecting onto $T$ along geodesics in $\mathrm{H}^{2}$ orthogonal to $T$. Note that all its fibers $g^{-1}(t), t \in T$ are isometric to $\mathbb{R}^{n+1}$ and geodesically embedded in $\mathrm{H}^{2} \times \mathbb{R}^{n}$. Composing $g$ with $f$ and identifying $T=\mathbb{R}$ we obtain a (smooth) function $h: \mathrm{H}^{3} \rightarrow \mathbb{R}$, $h=g \circ f$.

Next, we fix $y_{0} \in \mathrm{H}^{3}$ and consider the metric sphere $S_{R} \subset \mathrm{H}^{3}$ of a sufficiently large radius $R$ centered at $y_{0}$. Considered with the induced intrinsic metric, $S_{R}$ is isometric to the standard sphere $S_{\rho}^{2} \subset \mathbb{R}^{3}$ of radius $\rho$ which is exponentially large in $R, \rho \sim e^{R}$. We may further assume for 
simplicity that $h$ restricted to $S_{R}$ is a Morse function, $h: S_{\rho}^{2} \rightarrow \mathbb{R}$. Now we come to the crucial point. Every level set $\gamma_{t}=h^{-1}(t), t \in \mathbb{R}$ is mapped by $f$ into the $(n+1)$-flat $F=g^{-1}(t) \subset \mathrm{H}^{2} \times \mathbb{R}^{n}$. Since $f$ is Lipschitz and $F$ is geodesic, $f\left(\gamma_{t}\right)$ sits in a ball in $F$ of radius $\sim R$ with respect to the induced intrinsic metric on $F$. Thus $f\left(\gamma_{t}\right)$ has a polynomial in $R$ size, where by "size" we mean, for example, the minimal number of points of some separated net in $f\left(\mathrm{H}^{3}\right)$ needed to cover $f\left(\gamma_{t}\right)$ by balls of a fixed radius centered at these points. Since $f$ is biLipschitz, this implies that every connected component of $\gamma_{t} \subset S_{\rho}^{2}$ (but may be not the whole $\gamma_{t}$ !) has the diameter polynomial in $R$, i.e., essentially smaller than $\rho$. This conclusion is a key point which easily leads to a contradiction. Having all connected components of all level sets $\gamma_{t}$ sufficiently small compare to $\rho$ one can, for example, continuously contract the sphere $S_{\rho}^{2}$ to a point (with some little work near critical levels of index one). However, these details are inessential for the generalization and we omit them.

Acknowledgment. The first author is happy to express his deep gratitude to the University of Zürich for the support, hospitality and excellent working conditions while writing the paper.

\section{Subexponential corank}

In this section we define the subexponential corank of a metric space $X$ and establish some of its properties which are needed for the proof and applications of Theorem 1.1.

\subsection{Preliminaries}

The key notions of CAT(-1)-spaces and Hadamard spaces are well established by now, and the reader may consult, for instance, $[\mathrm{BH}]$ for them.

Given a metric space $X$ and $x \in X$, we denote by $B_{R}(x)$ the open ball of radius $R$ in $X$ centered at $x$ and by $\bar{B}_{R}(x)$ the corresponding closed ball. A subset $A \subset X$ is called net if $\operatorname{dist}(A, X)<\infty$. $A$ is $\delta$-separated, $\delta>0$, if $\operatorname{dist}\left(a, a^{\prime}\right) \geq \delta$ for every distinct $a, a^{\prime} \in A$. We usually use the notation $X_{\delta}$ for a separated net in $X$, where $\delta$ is the separation constant of $X_{\delta}$. Note that the balls $B_{\delta}(a)$ centered at the points $a \in X_{\delta}$ of some maximal separated net $X_{\delta} \subset X$ cover $X$.

Assume that a maximal separated net $X_{\delta} \subset X$ and $\sigma \geq \delta$ are fixed. Then we define the size of $A \subset X$ (w.r.t. $X_{\delta}$ and $\sigma$ ) as the number $\operatorname{size}_{X_{\delta}, \sigma}(A) \in$ $\mathbb{N} \cup\{\infty\}$ of points $x \in X_{\delta}$ with $B_{\sigma}(x) \cap A \neq \emptyset$. By the remark above, the union of all such balls contains $A$.

A map $f: X \rightarrow Y$ between metric spaces $X, Y$ is said to be quasiisometric, if

$$
\frac{1}{a} \operatorname{dist}\left(x, x^{\prime}\right)-b \leq \operatorname{dist}\left(f(x), f\left(x^{\prime}\right)\right) \leq a \operatorname{dist}\left(x, x^{\prime}\right)+b
$$


for some $a \geq 1, b \geq 0$ and all $x, x^{\prime} \in X$. In this case we say that $f$ is $(a, b)$-quasi-isometric. If in addition $f(X)$ is a net in $Y$ then $f$ is called $a$ quasi-isometry and the spaces $X$ and $Y$ are quasi-isometric.

Recall that the (topological) dimension of a compact set $K$ is the minimal integer $n=\operatorname{dim} K$ such that one can inscribe in every open covering of $K$ a finite closed covering having the multiplicity $\leq n+1$. If in addition $K$ is a metric space then $\operatorname{dim} K$ is the same as the minimal number $n$ having the property that for every $\varepsilon>0$ there is a finite closed covering of $K$ by sets with diameter $<\varepsilon$ and the multiplicity $\leq n+1$. For a topological space $X$ we use the definition $\operatorname{dim} X=\sup \{\operatorname{dim} K: K \subset X$ is compact $\}$.

\subsection{Definition of the subexponential corank}

A continuous partition

$$
X=\bigcup_{t \in T} g^{-1}(t)
$$

of a metric space $X$ is given by a continuous map $g: X \rightarrow T$. We use the notation $(X, g, T)$ and say that the partition $(X, g, T)$ has rank $k=$ $\sup \{\operatorname{dim} g(K): K \subset X$ is compact $\}$. Fix $x_{0} \in X$. A continuous partition $(X, g, T)$ is said to be subexponential if the following holds. For each maximal separated net $X_{\delta} \subset X$ with a sufficiently large separation constant $\delta$, each sufficiently large $\sigma \geq \delta$ and every $\varepsilon>0$ there exists $R_{0}=R_{0}\left(X_{\delta}, \sigma, \varepsilon\right) \geq 1$ such that for every $R \geq R_{0}$ and every $t \in T$ we have

$$
\frac{1}{R} \ln \operatorname{size}_{X_{\delta}, \sigma}\left(g^{-1}(t) \cap \bar{B}_{R}\left(x_{0}\right)\right)<\varepsilon .
$$

This property, obviously, is independent of the choice of $x_{0} \in X$. Now we define the subexponential corank of $X$ as

$$
\operatorname{corank} X=\sup _{Z \sim X} \inf \operatorname{rank}(Z, g, T) \text {, }
$$

where the supremum is taken over all $Z$ quasi-isometric to $X$ and the infimum is over all subexponential partitions of $Z$. Clearly, corank $X$ is a quasi-isometric invariant.

Remark 2.1. It is the controversy between continuity conditions needed for the proof of Theorem 1.1 and the quasi-isometry invariance needed for its applications which makes the definition of corank $X$ rather entangled. In particular, taking the supremum over all $Z$ quasi-isometric to $X$ in the definition of corank $X$ is necessary because for any discrete space $Z$ the trivial partition $(Z, \mathrm{id}, Z)$ is subexponential and has rank 0 , i.e., minimal possible rank.

Remark 2.2. The property of a partition $(X, g, T)$ to be subexponential means roughly speaking that every fiber $g^{-1}(t)$ has a subexponential growth 
rate and a bounded distortion in $X$. The last condition is essential. For example, all fibers of the partition $\left(\mathrm{H}^{n}, g, \mathbb{R}\right)$ given by a Busemann function $g: \mathrm{H}^{n} \rightarrow \mathbb{R}$ are isometric to $\mathbb{R}^{n-1}$ in the induced Riemannian metric and hence they have a subexponential (in fact, polynomial) growth rate. However, this partition is by no means subexponential because each horosphere $g^{-1}(t), t \in \mathbb{R}$ is exponentially distorted in $\mathrm{H}^{n}$ and for a fixed $x_{0} \in \mathrm{H}^{n}$ the balls $\bar{B}_{R}\left(x_{0}\right)$ contain exponentially large pieces of it.

We shall frequently use the following actually straightforward

Lemma 2.3. If $f: X \rightarrow Z$ is a continuous quasi-isometric map and $(Z, g, T)$ is a subexponential partition then $(X, g \circ f, T)$ is a subexponential partition too.

Proof. Assuming that $f$ is $(a, b)$-quasi-isometric, we fix $x_{0} \in X$ and put $z_{0}=$ $f\left(x_{0}\right)$. Let $\delta_{0}, \sigma_{0} \geq \delta_{0}$ be the separation and radius constants respectively for $Z$ involved in the subexponential size estimate. We put $\delta=a\left(\delta_{0}+b\right)$ and take a maximal separated net $X_{\delta} \subset X$. Then $f\left(X_{\delta}\right)$ is $\delta_{0}$-separated, hence, it is contained in some maximal separated net $Z_{\delta_{0}} \subset Z$.

Next, we fix $\sigma \geq \max \left\{\delta, \frac{1}{a}\left(\sigma_{0}-b\right)\right\}$ and take $t \in T$. If $B_{\sigma}(x)$ intersects $(g \circ f)^{-1}(t) \cap \bar{B}_{R}\left(x_{0}\right)$ for some $x \in X_{\delta}$ then $f\left(B_{\sigma}(x)\right)$ intersects $g^{-1}(t) \cap \bar{B}_{a R+b}\left(z_{0}\right)$ since $f\left(\bar{B}_{R}\left(x_{0}\right)\right) \subset \bar{B}_{a R+b}\left(z_{0}\right)$. Thus $B_{a \sigma+b}(f(x))$ intersects $g^{-1}(t) \cap \bar{B}_{a R+b}\left(z_{0}\right)$ and consequently we have

$$
\operatorname{size}_{X_{\delta}, \sigma}\left((g \circ f)^{-1}(t) \cap \bar{B}_{R}\left(x_{0}\right)\right) \leq \operatorname{size}_{Z_{\delta_{0}}, a \sigma+b}\left(g^{-1}(t) \cap \bar{B}_{a R+b}\left(x_{0}\right)\right)
$$

for every $R>0, t \in T$. Fix $\varepsilon>0$. Then for $a R+b \geq R_{0}\left(Z_{\delta_{0}}, a \sigma+b, \frac{\varepsilon}{a+b}\right)$ we obtain

$$
\frac{1}{a R+b} \ln \operatorname{size}_{X_{\delta}, \sigma}\left((g \circ f)^{-1}(t) \cap \bar{B}_{R}\left(x_{0}\right)\right)<\frac{\varepsilon}{a+b} .
$$

Thus taking $R_{0}\left(X_{\delta}, \sigma, \varepsilon\right) \geq \max \left\{1, \frac{1}{a}\left[R_{0}\left(Z_{\delta_{0}}, a \sigma+b, \varepsilon /(a+b)\right)-b\right]\right\}$ we obtain the required estimate

$$
\frac{1}{R} \ln \operatorname{size}_{X_{\delta}, \sigma}\left((g \circ f)^{-1}(t) \cap \bar{B}_{R}\left(x_{0}\right)\right)<\varepsilon
$$

for every $R \geq R_{0}\left(X_{\delta}, \sigma, \varepsilon\right)$ and $t \in T$.

Lemma 2.4. Assume that a metric space $X$ is quasi-isometric to a $Q P C$ space $Z$. Then

$$
\operatorname{corank} X=\inf \operatorname{rank}(Z, g, T),
$$

where the infimum is taken over all subexponential partitions of $Z$.

Proof. If $X^{\prime}$ is quasi-isometric to $X$ then it is quasi-isometric to $Z$ too. Any map $X^{\prime} \rightarrow Z$ parallel to a quasi-isometric one is quasi-isometric, thus there is a continuous quasi-isometry $X^{\prime} \rightarrow Z$. By Lemma 2.3 we have $\inf \operatorname{rank}\left(X^{\prime}, g^{\prime}, T^{\prime}\right) \leq \inf \operatorname{rank}(Z, g, T)$. Hence, the claim. 
Now we explain why all locally compact Hadamard spaces are QPC, though the argument (which is basically standard) might be applied to a much broader class of metric spaces.

Lemma 2.5. Every locally compact Hadamard space $X$ is a $Q P C$-space.

Proof. Assume that we have an $(a, b)$-quasi-isometric map $f: Y \rightarrow X$. We take a maximal $\delta$-separated net $Y_{\delta} \subset Y$ with $a \delta+b \geq \delta_{0}>0$ and note that every ball $B_{2 \delta}(\alpha)$ with $\alpha \in Y_{\delta}$ contains only a finite number of elements of $Y_{\delta}$. This is because $f\left(Y_{\delta}\right)$ is $\delta_{0}$-separated and $X$ is finitely compact, thus the ball $B_{2 a \delta+b}(f(\alpha)) \supset f\left(B_{2 \delta}(\alpha)\right)$ intersects $f\left(Y_{\delta}\right)$ over a finite set. It follows that the nerve $\mathcal{N}$ of the covering $\mathcal{A}=\left\{B_{\delta}(\alpha): \alpha \in Y_{\delta}\right\}$ of $Y$ is a locally finite simplicial complex. Choosing a continuous partition of unity $\left\{p_{\alpha}: Y \rightarrow \mathbb{R}: \alpha \in Y_{\delta}\right\}$ subordinate to $\mathcal{A}$, we obtain a continuous map $g: Y \rightarrow \mathcal{N}$ by

$$
g(y)=\sum_{\alpha \in Y_{\delta}} p_{\alpha}(y) \alpha,
$$

where we identify $Y_{\delta}$ with the 0 -skeleton of $\mathcal{N}$. Note that $g(y)$ lies in the simplex $\Delta_{y}$ spanned by $\left\{\alpha \in Y_{\delta}: \operatorname{dist}(\alpha, y)<\delta\right\}$.

Next, we extend $f \mid Y_{\delta}: \operatorname{ske}_{0} \mathcal{N} \rightarrow X$ to a continuous $\bar{f}: \mathcal{N} \rightarrow X$ using convexity of $X$ and acting by the induction on the dimension of the skeletons. Then $\bar{f}\left(\Delta_{y}\right) \subset B_{a \delta+b}(f(y))$ and thus $\bar{f} \circ g: Y \rightarrow X$ is a continuous map parallel to $f$.

\subsection{Properties of the subexponential corank}

We list some properties of corank which easily follow from the definition.

(1) $\operatorname{corank} X \leq \operatorname{dim} X$ for every QPC-space $X$. This immediately follows from Lemma 2.4.

(2) $\operatorname{corank}\left(\mathbb{R}^{n}\right)=0$ for each $n \geq 0$. Moreover, if the volume entropy

$$
h(X)=\lim \sup _{R \rightarrow 0} \frac{1}{R} \ln \operatorname{size}\left(B_{R}\left(x_{0}\right)\right)=0,
$$

then corank $X=0$. Note that the condition $h(X)=0$ is a quasi-isometry invariant. In this case, the trivial partition $(X, g,\{\mathrm{pt}\})$ given by a constant map $g$ is subexponential.

(3) $\operatorname{corank}\left(X_{1} \times X_{2}\right) \leq \operatorname{corank}\left(X_{1}\right)+\operatorname{corank}\left(X_{2}\right)$ if $X_{1} \times X_{2}$ is a QPCspace. In this case, both $X_{1}, X_{2}$ are QPC and the product partition $\left(X_{1} \times\right.$ $\left.X_{2}, g_{1} \times g_{2}, T_{1} \times T_{2}\right)$ of subexponential partitions $\left(X_{i}, g_{i}, T_{i}\right), i=1,2$ is subexponential. Finally, it is well known (see $[\mathrm{HW}])$ that $\operatorname{dim}(A \times B) \leq$ $\operatorname{dim} A+\operatorname{dim} B$ if at least one of the spaces $A, B$ is not empty.

(4) Let $X$ be a Hadamard manifold. Then $\operatorname{corank} X \leq \operatorname{dim} X-1$. Projecting onto a horosphere $T \subset X$ along geodesics orthogonal to $T$, we obtain the subexponential partition $(X, g, T)$ of $\operatorname{rank} \operatorname{dim} X-1$. Its fibers $g^{-1}(t)$ are geodesics. 
(5) Let $X$ be a Riemannian symmetric space of noncompact type. Then $\operatorname{corank} X \leq \operatorname{dim} X-\operatorname{rank} X$. An Iwasawa decomposition $G=N A K$ of the connected component of the identity in its isometry group allows to identify $X$ with the solvable group $N A$. Fix $x_{0} \in X$ and consider the orbit $T=N x_{0} \subset X$. Then the map $g: X \rightarrow T$ given by $g(x)=n x_{0}$, where $x=\operatorname{nax}_{0}$ for $n \in N, a \in A$, defines the subexponential partition $(X, g, T)$ of $\operatorname{rank} \operatorname{dim} T=\operatorname{dim} N=\operatorname{dim} X-\operatorname{rank} X$. Its fibers $g^{-1}(t)$ are geodesic rank $X$-flats.

(6) If $X$ is quasi-isometric to a QPC-space and $X^{\prime}$ admits a quasiisometric embedding into $X$ then corank $X^{\prime} \leq \operatorname{corank} X$. This follows Lemma 2.3 and Lemma 2.4.

\section{Hyperbolic rank and subexponential corank}

Here we prove Theorem 1.1 and its corollaries. The proof of Theorem 1.1 in outline goes as follows. By property (6) above it suffices to show that $\operatorname{rank}_{h} Y \leq \operatorname{corank} Y$ for each locally compact CAT $(-1)$ Hadamard space $Y$. Since $\operatorname{rank}_{h} Y=\operatorname{dim} \partial_{\infty} Y$ and $Y$ is certainly QPC it suffices to show that $\operatorname{dim} \partial_{\infty} Y \leq \operatorname{rank}(Y, g, T)$ for any subexponential partition $(Y, g, T)$.

We have a continuous map $\operatorname{pr}_{R}: \partial_{\infty} Y \rightarrow S_{R}$, where $S_{R} \subset Y$ is the metric sphere of radius $R$ centered at some fixed point $y_{0} \in Y$, given by the intersection of the ray $y_{0} \xi, \xi \in \partial_{\infty} Y$ with $S_{R}$. Using the CAT(-1) condition we introduce a metric $\mathrm{d}_{\infty}$ on $\partial_{\infty} Y$ for which $\mathrm{d}_{\infty}\left(\xi, \xi^{\prime}\right) \sim e^{-\operatorname{dist}\left(y_{0}, \xi \xi^{\prime}\right)}$ for $\xi$, $\xi^{\prime} \in \partial_{\infty} Y$, where $\xi \xi^{\prime}$ is the geodesic in $Y$ asymptotic to $\xi$ in one direction and to $\xi^{\prime}$ in the other one. Note that $\partial_{\infty} Y$ is compact since $Y$ is complete and locally compact and that the metric topology of $\mathrm{d}_{\infty}$ coincides with the topology of $\partial_{\infty} Y$. Thus the resulting map $h_{R}=g \circ \operatorname{pr}_{R}: \partial_{\infty} Y \rightarrow T$ is continuous and its image $K_{R}=h_{R}\left(\partial_{\infty} Y\right)$ is compact.

Now, we can inscribe in any open covering $\mathcal{O}$ of $K_{R}$ a finite closed covering $\mathcal{C}$ having the multiplicity $\leq n+1, n=\operatorname{rank}(Y, g, T)$. Since the partition $(Y, g, T)$ is subexponential, we may produce for each sufficiently large $R$ an open covering $\mathcal{O}_{R}$ of $K_{R}$ such that the size of the preimage in $S_{R}$ of every element of $\mathcal{O}_{R}$ is subexponential in $R$. Consequently, we can find a finite closed covering $\mathcal{C}_{R}$ of $K_{R}$ with the multiplicity $\leq n+1$ such that the size of the preimage in $S_{R}$ of every its element $C$ is subexponential in $R$. However, it does not mean that the diameter of $g^{-1}(C)$ measured in $S_{R}$ is subexponential in $R$ because it may have different connected components far away from each other. Nevertheless, we can rearrange the finite closed covering $\mathcal{A}_{R}=g^{-1}\left(\mathcal{C}_{R}\right)$ of $S_{R}$ in such a way that it is still finite, has the multiplicity $\leq n+1$ and the diameter of every its element is subexponential in $R$. In this way, we produce a finite closed covering $\mathcal{B}_{R}=\operatorname{pr}_{R}^{-1}\left(\mathcal{A}_{R}\right)$ of $\partial_{\infty} Y$ of multiplicity $\leq n+1$ with maximal diameter of its elements measured in the metric $\mathrm{d}_{\infty}$ going to 0 as $R \rightarrow \infty$. Hence, $\operatorname{dim} \partial_{\infty} Y \leq n$. Q.E.D. 
We fill in details answering the following questions

(1) how do we choose the open covering $\mathcal{O}_{R}$ of $K_{R}$;

(2) how to rearrange $\mathcal{A}_{R}$;

(3) how do we define the metric $d_{\infty}$;

(4) how to estimate $\operatorname{diam}(B)$ w.r.t. $\mathrm{d}_{\infty}$ for each $B \in \mathcal{B}_{R}$.

(1) Fix a maximal separated net $Y_{\delta} \subset Y$ and $\sigma \geq \delta$, which are sufficiently large to fit the $(Y, g, T)$ conditions, and a base point $y_{0} \in Y$. The notation $\operatorname{size}_{Y_{\delta}, \sigma}(A)$ will be abbreviated to $\operatorname{size}(A)$ for $A \subset Y$. Fix $\varepsilon$ with $0<\varepsilon<1$. For each $R>0$ and $t \in T$, the set $g^{-1}(t) \cap S_{R}\left(y_{0}\right)$ is covered by $N(R, t)=$ size $\left(g^{-1}(t) \cap S_{R}\left(y_{0}\right)\right)$ open balls $B_{\sigma}(y)$ with $y \in Y_{\delta}$. Using properties of the subexponential partition $(Y, g, T)$ we have

$$
\frac{1}{R} \ln N(R, t) \leq \frac{1}{R} \ln \operatorname{size}\left(g^{-1}(t) \cap \bar{B}_{R}\left(y_{0}\right)\right)<\varepsilon
$$

for every sufficiently large $R \geq 1$ and every $t \in T$.

Let $V \subset Y$ be the union of those balls. We claim that there is a neighborhood $U_{t}$ of $t$ with $g^{-1}\left(U_{t}\right) \cap S_{R}\left(y_{0}\right) \subset V$. Otherwise, we find a sequence $y_{i} \in S_{R}\left(y_{0}\right) \backslash V$ with $g\left(y_{i}\right) \rightarrow t$. Since $Y$ is complete and locally compact, we may assume that $y_{i} \rightarrow y_{\infty} \in S_{R}\left(y_{0}\right) \backslash V$. On the other hand, $y_{\infty} \in g^{-1}(t)$ by continuity of $g$. This is a contradiction.

Then $\mathcal{O}_{R}=\left\{U_{t}: t \in K_{R}\right\}$ is the required open covering of $K_{R}$ : we have

$$
\frac{1}{R} \ln \operatorname{size}\left(g^{-1}\left(U_{t}\right) \cap S_{R}\left(y_{0}\right)\right)<\varepsilon
$$

for each $t \in K_{R}$.

(2) For each $A \in \mathcal{A}_{R}$ we consider its covering by balls $B_{\sigma}(y), y \in Y_{\delta}$ which intersect $A$. The union of these balls has only a finite number of connected components $\alpha$ since every such $\alpha$ contains at least one ball $B_{\sigma}(y)$ and there is only a finite number of such balls in $B_{R+\sigma}\left(y_{0}\right)$. Now, we decompose $A$ into the finite disjoint union $A=\cup_{\alpha} A_{\alpha}$, where each $A_{\alpha}$ is the intersection of $A$ with a connected component $\alpha$. Thus the diameter of $A_{\alpha}$ measured in $S_{R}$ is at most $2 \delta \operatorname{size}(A), \operatorname{diam}_{R}\left(A_{\alpha}\right) \leq 2 \delta e^{\varepsilon R}$, and this gives the desired rearrangement of $\mathcal{A}_{R}$ (for which we use the same notation).

(3) We define the metric $\mathrm{d}_{\infty}$ on $\partial_{\infty} Y$ as follows. Given $\xi, \xi^{\prime} \in \partial_{\infty} Y$, we consider the unit speed geodesic rays $c_{\xi}, c_{\xi^{\prime}}$ from $y_{0}$ to $\xi, \xi^{\prime}$ respectively and put

$$
\mathrm{d}_{\infty}\left(\xi, \xi^{\prime}\right)=\lim _{s \rightarrow \infty} \angle\left(\overline{c_{\xi}}(s) \bar{y}_{0} \overline{\bar{c}_{\xi^{\prime}}}(s)\right),
$$

where $\angle\left(\overline{c_{\xi}}(s) \bar{y}_{0} \overline{c_{\xi^{\prime}}}(s)\right)$ is the angle at $\bar{y}_{0}$ of the comparison triangle in $\mathrm{H}^{2}$ for the triangle $c_{\xi}(s) y_{0} c_{\xi^{\prime}}(s)$. From the hyperbolic geometry we know that

$$
\tan \left(\frac{1}{4} \mathrm{~d}_{\infty}\left(\xi, \xi^{\prime}\right)\right)=e^{-\operatorname{dist}\left(\bar{y}_{0}, \overline{\xi \xi}^{\prime}\right)} .
$$


Thus $\mathrm{d}_{\infty}\left(\xi, \xi^{\prime}\right) \leq 4 e^{-\operatorname{dist}\left(y_{0}, \xi \xi^{\prime}\right)}$.

(4) Given $B \in \mathcal{B}_{R}$ let $\xi, \xi^{\prime} \in B$ be two remotest points (w.r.t. $\mathrm{d}_{\infty}$ ), $v=$ $\operatorname{pr}_{R}(\xi), v^{\prime}=\operatorname{pr}_{R}\left(\xi^{\prime}\right) \in S_{R}$. Then $v, v^{\prime} \in A$ for $A \in \mathcal{A}_{R}$ with $\operatorname{pr}_{R}^{-1}(A)=B$. In particular, $\operatorname{diam}_{R}(A) \leq 2 \delta e^{\varepsilon R}$. We take the midpoint $y$ of the geodesic segment $v v^{\prime} \subset Y$ and put $\rho=\operatorname{dist}\left(y_{0}, y\right), r=\operatorname{dist}\left(y, S_{R}\right)$. Then $\rho+r \geq R$, and an easy comparison argument shows that the length of any curve in $S_{R}$ between $v, v^{\prime}$ and hence $\operatorname{diam}_{R}(A)$ is at least as large as the length of a half-circle of radius $r$ in $\mathrm{H}^{2}$ (we use here that the points $v, v^{\prime}$ are visible from $y$ at the angle $\pi$ ). Thus we obtain $r \leq \varepsilon R+c$ for some $c=c(\delta)$. It follows

$$
\operatorname{dist}\left(y_{0}, \xi \xi^{\prime}\right) \geq \rho \geq R-r \geq(1-\varepsilon) R-c .
$$

Since $\varepsilon<1$, we have

$$
\operatorname{diam}(B)=\mathrm{d}_{\infty}\left(\xi, \xi^{\prime}\right) \leq 4 e^{c} e^{-(1-\varepsilon) R} \rightarrow 0 \quad \text { as } \quad R \rightarrow \infty .
$$

This completes the proof of Theorem 1.1.

Proof of Corollary 1.2. (1) By Lemma 2.5, $X$ is QPC. Thus corank $X \leq$ $\sum_{i=1}^{k} \operatorname{corank} X_{i} \leq \sum_{i=1}^{k}\left(\operatorname{dim} X_{i}-1\right)$ by Properties (3), (4), sect. 2. On the other hand, it follows from [FS] that $\operatorname{rank}_{h} X \geq \sum_{i=1}^{k} \operatorname{rank}_{h} X_{i}=$ $\sum_{i=1}^{k}\left(\operatorname{dim} X_{i}-1\right)$. By Theorem 1.1, $\operatorname{rank}_{h} X \leq \operatorname{corank} X$. Hence, the claim.

(2) We have $\operatorname{dim} X-\operatorname{rank} X \leq \operatorname{rank}_{h} X$ by [Le], $\operatorname{corank} X \leq \operatorname{dim} X-$ $\operatorname{rank} X$ by Property (5), and $\operatorname{rank}_{h} X \leq \operatorname{corank} X$ by Theorem 1.1. Hence, the claim.

\section{An exotic subexponential partition}

Here we prove Theorem 1.4. Recall (see, for instance, [BS, CK, KL]) that $X$ can be represented as the countable union $X=\cup_{v} X_{v}$ of blocks, where each $X_{v}$ is a closed convex subset in $X$ isometric to the metric product $F_{v} \times \mathbb{R}$ and $F_{v}$ is the universal covering of a compact nonpositively curved surface with geodesic boundary. Every two blocks are either disjoints or intersect over a boundary component which is a 2-flat in $X$ separating them and consequently no three blocks have a point in common. Furthermore, without loss of generality, we may assume that the Gaussian curvature of every $F_{v}$ is constant, $K \equiv-1$, and thus $F_{v}$ can be viewed as a closed convex subset in $\mathrm{H}^{2}$ bounded by countable many geodesic lines.

By Lemma 2.4, it suffices to construct a subexponential partition $(X, g, T)$ of rank 1. Actually, there are several ways to do that, for example, there is such an $(X, g, T)$ with $T=\mathbb{R}$ and $g^{-1}(t), t \in T$, homeomorphic to $\mathbb{R}^{2}$ and quasi-isometric to a subset of a template introduced in [CK]. This easily implies that each $g^{-1}(t)$ has a subexponential growth rate, however, to show that it has a bounded distortion in $X$ is not that easy and it requires 
a large piece of machinery from [CK]. Apparently, this difficulty is rooted in the fact that the target space $T=\mathbb{R}$ is in a sense simplest possible.

We shall construct another subexponential partition $(X, g, T)$ of rank 1 with $T$ much more complicated for which it is not immediately obvious even that $\operatorname{dim} T=1$. However, it is easy to establish the subexponential property of $(X, g, T)$ as well as the fact that $\operatorname{rank}(X, g, T)=1$.

To start with, we take a block $X_{v}=F_{v} \times \mathbb{R}$, fix a point $p \in F_{v}$ and $\rho>0$ with $\operatorname{dist}\left(p, \partial F_{v}\right)>2 \rho$ and consider the metric projection $\operatorname{pr}_{v}: F_{v} \backslash B_{2 \rho}(p) \rightarrow$ $S_{2 \rho}(p)$. Then $J_{w}=\operatorname{pr}_{v}\left(\partial_{w} F_{v}\right)$ is an open interval in the circle $S_{2 \rho}(p)$ for every connected component $\partial_{w} F_{v} \subset \partial F_{v}$.

Next, we note that $I_{w}=\operatorname{pr}_{v}^{-1}\left(\bar{J}_{w}\right) \subset F_{v}$ has an subexponential growth rate because its area is finite, $\operatorname{Area}\left(I_{w}\right)<\pi$ by Gauss-Bonnet. Now, we collaps every $\bar{J}_{w}$ to a point, $\eta: S_{2 \rho}(p) \rightarrow S$, and identify the resulting factor-space $S=S_{2 \rho}(p) / \sim$ with the circle $S=S_{\rho}(p) \subset F_{v}$. Furthermore, we may choose the identification in such a way that the geodesic segment $x \bar{x}$ lies in $\bar{B}_{2 \rho}(p) \backslash B_{\rho}(p)$ for every $x \in S_{2 \rho}(p)$ and its image $\bar{x} \in S$, and different such segments have no common interior points. Thus we can extend $\eta$ to the closed annulus $\bar{B}_{2 \rho}(p) \backslash B_{\rho}(p)$ by $\eta\left(x^{\prime}\right)=\bar{x}$ for each $x^{\prime} \in x \bar{x}$. Then $\left(F_{v} \backslash B_{\rho}(p), \eta \circ \mathrm{pr}_{v}, S\right)$ is a subexponential partition because $\left(\eta \circ \mathrm{pr}_{v}\right)^{-1}(x)$ is either a geodesic ray or some subset $I_{w}$. Note that there is only countable many points $x \in S$ for which the last case occurs. Any such point is said to be distinguished. Clearly, the set of the distinguished points is a countable dense subset in $S$.

Though the partition $\left(F_{v} \backslash B_{\rho}(p), \eta \circ \mathrm{pr}_{v}, S\right)$ has rank 1, we need to make further identifications to extend it on the whole $F_{v}$. To this end, we fix a diameter $T_{v} \subset \bar{B}_{\rho}(p)$ and take the metric projection $\bar{B}_{\rho}(p) \rightarrow T_{v}$. To avoid unnecessary complifications, we slightly perturb it in such a way that any two distinguished points of $S=\partial \bar{B}_{\rho}(p)$ are mapped into distinct points and images of at most two points of $S$ coincide. The images of the distinguised points are called distinguished too. Let $\zeta: \bar{B}_{\rho}(p) \rightarrow T_{v}$ be the perturbed map. Then $\left(F_{v}, \zeta \circ \eta \circ \mathrm{pr}_{v}, T_{v}\right)$ is a subexponential partition of rank 1 . From now on, we consider $T_{v}$ as an abstract space and not as a subset of $F_{v}$. Let $D_{v} \subset T_{v}$ be the subset of all distinguished points. Then $\zeta \circ \eta \circ \mathrm{pr}_{v}$ maps bijectively the set $\left\{\partial_{w} F_{v}\right\}$ of the boundary components of $F_{v}$ onto $D_{v}$.

Coming back to the block $X_{v}=F_{v} \times \mathbb{R}$, we compose the projection on the first factor with $\zeta \circ \eta \circ \mathrm{pr}_{v}$ and denote the resulting map by $g_{v}: X_{v} \rightarrow T_{v}$. Then, obviously, $\left(X_{v}, g_{v}, T_{v}\right)$ is a subexponential partition of rank 1 , and $g_{v}$ maps the set of the boundary components of $X_{v}$ bijectively onto $D_{v}$.

Finally, let $\widetilde{T}=\sqcup_{v} T_{v}$ be the disjoint union. We define $T:=\widetilde{T} / \sim$, where distinct $t \in T_{v}, t^{\prime} \in T_{v^{\prime}}$ are equivalent if and only if $t \in D_{v}, t^{\prime} \in D_{v^{\prime}}$, the blocks $X_{v}, X_{v^{\prime}}$ are different and adjacent along a common boundary component which corresponds to $t$ and $t^{\prime}$. Then $\widetilde{g}=\sqcup_{v} g_{v}: \sqcup_{v} X_{v} \rightarrow \sqcup_{v} T_{v}$ factors to a continuous $g: X \rightarrow T$, and the partition $(X, g, T)$ is subexponential: for every distinguished $t \in T$, the set $g^{-1}(t)$ sits in two adjacent blocks and 
it is (outside of a $\{$ compact set $\} \times \mathbb{R}$ ) the union of a geodesic half-plane and a subexponential $I_{w} \times \mathbb{R}$ in each of the blocks. Otherwise, $g^{-1}(t)$ is (again outside of a $\{$ compact set $\} \times \mathbb{R}$ ) the union of two geodesic half-planes sitting in one and the same block. Each compact set $K \subset X$ intersects only finitely many blocks $X_{v}$, hence its image $g(K) \subset T$ intersects only finitely many segments $T_{v}$ and consequently $\operatorname{dim} g(K) \leq 1$. Thus $\operatorname{rank}(X, g, T)=1$. This completes the proof of Theorem 1.4.

\section{References}

[BF] N. Brady, B. Farb, Filling-invariants at infinity for manifolds of nonpositive curvature, Trans. Amer. Math. Soc, 350 (1998), 3393-3405.

[BH] M. Bridson, A. Haefliger, Metric spaces of nonpositive curvature, Grundlehren der Mathematischen Wissenschaften, 319. SpringerVerlag, Berlin, 1999.

[BS] S. Buyalo, V. Schroeder, On the asymptotic geometry of nonpositively curved graphmanifolds, Trans. Amer. Math. Soc (to appear).

[CK] C. Croke, B. Kleiner, Geodesic flow on a nonpositively curved graph manifold.

[FS] T. Foertsch, V. Schroeder, Hyperbolic rank of products, in preparation.

[Gr] M. Gromov, Asymptotic invariants of infinite groups, in "Geometric Group Theory", (G.A. Niblo, M.A. Roller, eds.), London Math. Soc. Lecture Notes Series 182 Vol. 2, Cambridge University Press, 1993.

[HW] W. Hurevicz, H. Wallman, Dimesion Theory, Princeton, Princeton University Press, 1948.

[KL] M. Kapovich, B. Leeb, 3-manifold groups and nonpositive curvature, Geom. funct. anal. 8 (1998), 841-852.

[Le] E. Leuzinger, Corank and asymptotic filling-invariants for symmetric spaces, Geom. funct. anal. 10 (2000), 863-873.

Sergei Buyalo, Steklov Institute of Mathematics, Fontanka 27, 191011, St. Petersburg, Russia

buyalo@pdmi.ras.ru

Viktor Schroeder, Institut für Mathematik, Universität Zürich, Winterthurer Strasse 190, CH-8057 Zürich, Switzerland

vschroed@math.unizh.ch 